\title{
Neutrinoless double beta decay within Self-consistent Renormalized Quasiparticle Random Phase Approximation and inclusion of induced nucleon currents
}

\author{
A. Bobyk, W. A. Kamiński \\ Department of Theoretical Physics, Maria Curie-Sktodowska University, \\ Radziszewskigo 10, PL-20-031 Lublin, Poland. \\ F. Šimkovic \\ Department of Nuclear Physics, Comenius University, \\ Mlynska dol., pav. F1, SK-842 15 Bratislava, Slovakia
}

(October 27, 2018)

\begin{abstract}
The first, to our knowledge, calculation of neutrinoless double beta decay $(0 \nu \beta \beta$-decay $)$ matrix elements within the self-consistent renormalized Quasiparticle Random Phase Approximation (SRQRPA) is presented. The contribution from the momentum-dependent induced nucleon currents to $0 \nu \beta \beta$ decay amplitude is taken into account. A detailed nuclear structure study includes the discussion of the sensitivity of the obtained SRQRPA results for $0 \nu \beta \beta$-decay of ${ }^{76}$ Ge to the parameters of nuclear Hamiltonian, two-nucleon short-range correlations and the truncation of the model space. A comparison with the standard and renormalized QRPA results is presented. We have found a considerable reduction of the SRQRPA nuclear matrix elements, resulting in less stringent limits for the effective neutrino mass. PACS numbers: 23.40.Hc, 21.60.Jz, 27.50.+e, 27.60.+j
\end{abstract}

The neutrino mass and mixing of neutrinos are the main subject of the elementary 
particle physics nowadays. The experiments looking for oscillation of solar, atmospheric and terrestrial (LSND-experiment) neutrinos constitute evidence for a new physics beyond the Standard Model [1]. The current constraints imposed by the results of neutrino oscillation experiments allow to construct the spectrum of mass of neutrinos [2]. The predictions differ from each other by different input, structure of neutrino mixing and assumption, e.g., on the phases and fundamental character of neutrinos.

An important quantity to limit the space of possible neutrino mixing schemes is the effective Majorana neutrino mass

$$
\left\langle m_{\mathrm{ee}}\right\rangle=\left|\sum_{k}\left(U_{\mathrm{e} k}\right)^{2} m_{k} \eta_{k}^{\mathrm{CP}}\right|
$$

where $U_{\mathrm{e} k}, m_{k}$ and $\eta_{k}^{\mathrm{CP}}$ are unitary mixing matrix elements, mass eigenstates and the relative CP-phases of neutrinos, respectively. The value of $\left\langle m_{\mathrm{ee}}\right\rangle$ depends on the specific model of neutrino mixing and its predictions fall in the range of $10^{-3} \mathrm{eV}$ to few eV [2]. The upper bound on $\left\langle m_{\mathrm{ee}}\right\rangle$ can be deduced from the current experimental lower bound on the half-life of neutrinoless double beta decay $(0 \nu \beta \beta$-decay) as follows [3 5 :

$$
\left\langle m_{\mathrm{ee}}\right\rangle \leq \frac{m_{\mathrm{e}}}{\left|M_{\left\langle m_{\mathrm{ee}}\right\rangle}\right| \sqrt{T_{1 / 2}^{0 \nu-\exp G_{01}}}}
$$

Here, $m_{\mathrm{e}}, G_{01}$ and $M_{\left\langle m_{\mathrm{ee}}\right\rangle}$ are the mass of electron, the integrated kinematical factor [3, 10] and the $0 \nu \beta \beta$-decay nuclear matrix element, respectively.

The present most stringent lower bound on $T_{1 / 2}^{0 \nu-\exp }$ has been measured for ${ }^{76} \mathrm{Ge}$ by Heidelberg-Moscow group and is equal to $5.7 \times 10^{25}$ years [6,7]. A condition for obtaining reliable limit for fundamental particle physics quantity $\left\langle m_{\mathrm{ee}}\right\rangle$ is that the nuclear matrix elements governing the Majorana mass mechanism of $0 \nu \beta \beta$-decay can be calculated correctly [5]. However, the practical nuclear structure calculation always involves some approximations, which make it difficult to obtain an unambiguous limit on $\left\langle m_{\mathrm{ee}}\right\rangle$. We note that the difference between the previous $\left(M_{\left\langle m_{\mathrm{ee}}\right\rangle}=4.18\right.$, i.e., $\left.\left\langle m_{\mathrm{ee}}\right\rangle \leq 0.18\right)$ [4] and the recent more advanced $\left(M_{\left\langle m_{\mathrm{ee}}\right\rangle}=1.67\right.$, i.e., $\left.\left\langle m_{\mathrm{ee}}\right\rangle \leq 0.46\right)[$ shell model calculations is significant. There is also an other group of nuclear structure calculations which include the proton-neutron 
Quasiparticle Random Phase Approximation (pn-QRPA) [9] and its extensions [10,11]. Some of them suggest the upper bound on $\left\langle m_{\mathrm{ee}}\right\rangle$ to be in the $0.1 \mathrm{eV}$ range [6].

The aim of the present letter is to discuss the nuclear physics aspects of the pn-QRPA [9], the renormalized pn-QRPA (pn-RQRPA) [12,13] and the self-consistent pn-RQRPA (pn-SRQRPA) 16,17 calculation of the nuclear matrix element $M_{\left\langle m_{\mathrm{ee}}\right\rangle}$ for $0 \nu \beta \beta$-decay of ${ }^{76} \mathrm{Ge}$. We note that the pn-SRQRPA results on $0 \nu \beta \beta$-decay matrix elements have been not presented in the literature till now.

We shortly present the main differences between the above mentioned three QRPA approaches.

The pn-QRPA has been the most popular theoretical tool in description of the $\beta$ and $\beta \beta$ decays of medium and heavy open shell nuclei. However, the pn-QRPA develops a collapse beyond some critical value of the particle-particle interaction strength of nuclear Hamiltonian close to its realistic value. This phenomena makes predictive power of the obtained results questionable.

By implementing the Pauli exclusion principle (PEP) in an approximate way in the pnQRPA one gets the pn-RQRPA [12,13], which avoids collapse within a physical range of particle-particle force and offers more stable solution. This fact has been confirmed also within the schematic models. In addition it was found that by restoring the PEP a better agreement with the exact solution is obtained [14].

The selfconsistent pn-RQRPA (pn-SRQRPA) [15,17, 16], which is more complex version of the RQRPA, is becoming increasingly popular to describe strongly correlated Fermion systems. The pn-SRQRPA goes a step further beyond the pn-RQRPA. In the pn-SRQRPA at the same time the mean field is changed by minimizing the energy and fixing the number of particles in the correlated ground state instead of the uncorrelated BCS one as it is done in the other versions of the QRPA (pn-QRPA, pn-RQRPA) 15, 17, 16. Thus the pn-SRQRPA is closer to a fully variational theory.

We proceed by writing the expression for the $0 \nu \beta \beta$-decay nuclear matrix element. We note that the contribution to $M_{\left\langle m_{\mathrm{ee}}\right\rangle}$ from the induced pseudoscalar term of the nucleon 
current were not considered before. Recently, it has been found that it is significant and leads to a modification of Gamow-Teller and new tensor contributions of $\left.M_{\left\langle m_{\mathrm{ee}}\right\rangle} \llbracket 11\right]$. Thus $M_{\left\langle m_{\mathrm{ee}}\right\rangle}$ is given as sum of Fermi, Gamow-Teller and tensor contributions

$$
M_{\left\langle m_{\mathrm{ee}}\right\rangle}=-\frac{M_{F}}{g_{A}^{2}}+M_{G T}+M_{T}
$$

with $g_{A}=1.25$. Expressed in relative coordinates and using the second quantization formalism $M_{\left\langle m_{\mathrm{ee}}\right\rangle}$ takes the form

$$
\begin{aligned}
M_{\left\langle m_{\mathrm{ee}}\right\rangle}= & \sum_{J^{\pi}} \sum_{\substack{p n p^{\prime} n^{\prime} \\
m_{i} m_{f} \mathcal{J}}}(-)^{j_{n}+j_{p^{\prime}}+J+\mathcal{J}}(2 \mathcal{J}+1)\left\{\begin{array}{ccc}
j_{p} & j_{n} & J \\
j_{n^{\prime}} & j_{p^{\prime}} & \mathcal{J}
\end{array}\right\} \\
& \times\left\langle p(1), p^{\prime}(2) ; \mathcal{J}\left|f\left(r_{12}\right) \tau_{1}^{+} \tau_{2}^{+} \mathcal{O}_{\left\langle m_{\mathrm{ee}}\right\rangle}(12) f\left(r_{12}\right)\right| n(1), n^{\prime}(2) ; \mathcal{J}\right\rangle \\
& \times\left\langle 0_{f}^{+}\left\|\left[c_{p^{\prime}}^{+} \tilde{c}_{n^{\prime}}\right] J\right\| J^{\pi} m_{f}\right\rangle\left\langle J^{\pi} m_{f} \mid J^{\pi} m_{i}\right\rangle\left\langle J^{\pi} m_{i}\left\|\left[c_{p}^{+} \tilde{c}_{n}\right]_{J}\right\| 0_{i}^{+}\right\rangle .
\end{aligned}
$$

Here, $f\left(r_{12}\right)$ is the short-range correlation function and $\mathcal{O}_{\left\langle m_{\mathrm{ee}}\right\rangle}(12)$ represents the coordinate and spin dependent part of the two-body $0 \nu \beta \beta$-decay transition operator

$$
\mathcal{O}_{\left\langle m_{\mathrm{ee}}\right\rangle}(12)=-\frac{H_{F}\left(r_{12}\right)}{g_{A}^{2}}+H_{G T}\left(r_{12}\right) \sigma_{12}+H_{T}\left(r_{12}\right) \mathbf{S}_{12}
$$

where $\sigma_{12}=\vec{\sigma}_{1} \cdot \vec{\sigma}_{2}, S_{12}=3\left(\vec{\sigma}_{1} \cdot \hat{q} \vec{\sigma}_{2} \cdot \hat{q}\right)-\sigma_{12}$, and

$$
H_{K}\left(r_{12}\right)=\frac{2}{\pi g_{A}^{2}} \frac{R}{r_{12}} \int_{0}^{\infty} \frac{\sin \left(q r_{12}\right)}{q+E^{m}(J)-\left(E^{i}+E^{f}\right) / 2} h_{K}\left(q^{2}\right) d q,(K=F, G T, T)
$$

with

$$
\begin{aligned}
h_{F}\left(q^{2}\right) & =g_{V}^{2}\left(q^{2}\right) g_{A}^{2} \\
h_{G T}\left(q^{2}\right) & =g_{A}^{2}\left(q^{2}\right)+\frac{1}{3} \frac{g_{P}^{2}\left(q^{2}\right) q^{4}}{4 m_{p}^{2}}-\frac{2}{3} \frac{g_{A}\left(q^{2}\right) g_{P}\left(q^{2}\right) q^{2}}{2 m_{p}} \\
h_{T}\left(q^{2}\right) & =\frac{2}{3} \frac{g_{A}\left(q^{2}\right) g_{P}\left(q^{2}\right) q^{2}}{2 m_{p}}-\frac{1}{3} \frac{g_{P}^{2}\left(q^{2}\right) q^{4}}{4 m_{p}^{2}} .
\end{aligned}
$$

Here, $R$ is the mean nuclear radius [11]. $E^{i}, E^{f}$ and $E^{m}(J)$ are respectively the energies of the initial, final and intermediate nuclear state with angular momentum $J$. The momentum dependence of the vector, axial-vector and pseudoscalar formfactors $\left(g_{V}\left(q^{2}\right), g_{A}\left(q^{2}\right)\right.$ and $\left.g_{P}\left(q^{2}\right)\right)$ is given in Ref. [11. 
We note that the first sum on the r.h.s of Eq. (4) represents summation over all multipolarities. The form of the one-body transition densities [see Eq. (4) ] to excited states $\left|J^{\pi} m_{i}\right\rangle$ and $\left|J^{\pi} m_{f}\right\rangle$ generated from the initial $(\mathrm{A}, \mathrm{Z})$ and the final $(\mathrm{A}, \mathrm{Z}+2)$ ground states $\left|0_{i}^{+}\right\rangle$and $\left.0_{f}^{+}\right\rangle$within the pn-RQRPA and the pn-SRQRPA is the same and can be found together with other details of the nuclear structure in Refs. [13, 11, 17]. The difference consists only in the calculated value of the renormalized coefficients $D$, which in the case of the pn-QRPA is just equal to unity. The overlap factor entering the expression (4) can be find in Ref. [18].

The calculation of $0 \nu \beta \beta$-decay matrix elements of ${ }^{76} \mathrm{Ge}$ are performed within two model spaces both for protons and neutrons as follows: i) The model space I (m.s. I) consists of the full $3-4 \hbar \omega$ major oscillator shells and has been considered in the pn-QRPA studies of Ref. [9] (9-levels model space). ii) The model space II (m.s. II) comprises the full $2-5 \hbar \omega$ major shells (12 levels model space).

The single particle energies were obtained by using a Coulomb-corrected Woods-Saxon potential with Bertsch parameterization. Two-body G-matrix elements were calculated from the Bonn one-boson exchange potential within within the Brueckner theory. In the pn-QRPA and the pn-RQRPA approaches pairing interactions have been adjusted to fit the empirical pairing gaps. In the pn-SRQRPA approach the pairing matrix elements of the NN interaction have not been rescaled as the mean field is directly related to the excited states. The particle-particle and particle-hole channels of the G-matrix interaction of the nuclear Hamiltonian $H$ are renormalized by introducing the parameters $g_{\mathrm{pp}}$ and $g_{\mathrm{ph}}$, respectively.

In Fig. 11 the calculated partial matrix elements $M_{F}, M_{G T}$ and $M_{T}$ are plotted as function of $g_{\mathrm{pp}}\left(g_{\mathrm{ph}}=1.0\right)$ for the pn-RQRPA and pn-SRQRPA approaches. The larger 12-levels model space is considered. One find a strong dependence of $M_{F}$ and $M_{G T}$ on $g_{\mathrm{pp}}$. The smallest $M_{T}$ matrix element is rather insensitive to this parameter. In general, the behavior of plotted matrix elements is similar for both approaches. Nevertheless, the Fermi and GT matrix elements of the pn-SRQRPA one reach zero value inside the physical range of $g_{\mathrm{pp}}$ parameter $\left(0.8 \leq g_{\mathrm{pp}} \leq 1.2\right)$.

In Fig. 2 the multipole decomposition (according to intermediate multipoles $J^{\pi}$ ) of 
matrix element $M_{\left\langle m_{\mathrm{ee}}\right\rangle}$ for the pn-RQRPA and pn-SRQRPA approaches and two values of $g_{\mathrm{pp}}\left(g_{\mathrm{pp}}=0.0\right.$ and $\left.g_{\mathrm{pp}}=1.0\right)$ is presented. The model space and $g_{\mathrm{ph}}$ is the same as in Fig. 1. The filled and open bars represent the calculations with and without consideration of the two-nucleon short-range correlations (s.r.c.), respectively. One clearly sees that the s.r.c. suppress the contributions from higher multipolarities. It just proves that the Majorana neutrino mass exchange mechanism is predominantly long-range interaction in the nucleus. We notice also that the contributions associated with even parity multipolarities are more influenced by the change of $g_{\mathrm{pp}}$ (especially in the case of the pn-SRQRPA) as those associated with the negative ones. The differences between the even parity multipole contributions calculated via the pn-RQRPA and the pn-SQRPA approaches is remendeous for $g_{\mathrm{pp}}=1.0$. This effect can be attributed to the different structure of the mean field in both approaches.

In Fig. [3 the nuclear matrix elements $M_{\left\langle m_{\mathrm{ee}}\right\rangle}$ as function of particle-particle strength $g_{\mathrm{pp}}$ for all three QRPA approaches (pn-QRPA, pn-RQRPA, pn-SRQRPA) is shown. One notices that the pn-RQRPA and the pn-SRQRPA results are much less sensitive to the truncation of model space and $g_{\mathrm{pp}}$ as the pn-QRPA ones. The unpleasant feature of the pn-QRPA and the pn-SRQRPA is that for the larger model space $M_{\left\langle m_{\mathrm{ee}}\right\rangle}$ crosses zero within the physical interval of $g_{\mathrm{pp}}$ what increase uncertainty in respect to the extracted value on $\left\langle m_{\mathrm{ee}}\right\rangle$ from non-observability of $0 \nu \beta \beta$-decay considerably.

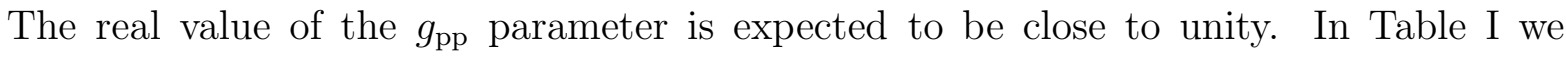
present $M_{\left\langle m_{\mathrm{ee}}\right\rangle}$ for this value of $g_{\mathrm{pp}}$ parameter together with the corresponding upper bound on $\left\langle m_{\mathrm{ee}}\right\rangle$ deduced from the Heisenberg-Moscow experiment. One notices that only the pn-QRPA results are sensitive to the truncation of model space. The new calculated pnSRQRPA results predict considerably larger value for upper limit on $\left\langle m_{\mathrm{ee}}\right\rangle$ in comparison with the pn-QRPA and the pn-RQRPA ones. It is due to the mean-field modifications by the number self-consistent approach which use to diminish the pairing gap [19].

In summary, the nuclear matrix element associated with the light Majorana neutrino mass mechanism of $0 \nu \beta \beta$-decay of ${ }^{76}$ Ge has been calculated in the selfconsistent pn-RQRPA approach which received much attention recently. This approach predicts considerably sup- 
pressed $0 \nu \beta \beta$-decay matrix element, what, when confronted with the present experimental data, leads to a less stringent limit on the effective Majorana neutrino mass parameter $\left\langle m_{\mathrm{ee}}\right\rangle$. The reduction of the matrix element is due to the modification of the mean field by the combined selfconsistent BCS+RQRPA approach which influences mostly the even parity multipole contributions.

This work was supported in part by the State Committee for Scientific Researches (Poland), Contract No. 2 P03 B00516. 


\section{REFERENCES}

[1] K. Zuber, Phys. Rep. 305, 295 (1988); S.M. Bilenky, C. Giunty, W. Grimus, Prog. Nucl. Part. Phys. 43, 1 (1999).

[2] S.M. Bilenky, C. Giunti, W. Grimus, B. Kayser, and S.T. Petcov, Phys. Lett. B 465, 193 (1999); F. Vissani, JHEP 9906, 22 (1999), and hep-ph/9906525; O. Haug, J.D. Vergados, Amand Faessler, and S. Kovalenko, Nucl. Phys. B 565, 38 (2000); A. Kalliomaki and J. Maalampi, Phys. Lett. B 484, 64 (2000); H.V. Klapdor-Kleingrothaus, H. Päs, A.Y. Smirnov, hep-ph/0003219. W. Rodejohann, hep-ph/0008044.

[3] M. Doi, T. Kotani, and E. Takasugi, Prog. Theor. Phys. (Supp.) 83, 1 (1985).

[4] W.C. Haxton, and G.J. Stephenson, Prog. Part. Nucl. Phys. 12, 409 (1984)

[5] A. Faessler and F. Šimkovic, J. Phys. G 24, 2139 (1998); J. Suhonen and O. Civitarese, Phys. Rep. 300, 123 (1998); J.D. Vergados, Phys. At. Nucl. 63, 1137 (2000); R.N. Mohapatra, Nucl. Phys. B, Proc. Suppl. 77, 376 (1999); P.Vogel, nucl-th/0005020.

[6] L. Baudis et al., Phys. Rev. Lett. 83, 41 (1999);

[7] F.T. Avignone III, C. E. Aalseth, R. L.Brodzinski, Phys. Rev. Lett. 85, 465 (2000).

[8] E. Caurier, F. Nowacki, A. Poves, and J. Retamosa, Phys. Rev. Lett. 77, 1954 (1996).

[9] P. Vogel and M.R. Zirnbauer, Phys. Rev. Lett. 148, 3148 (1986); O. Civitarese, A. Faessler, and T. Tomoda, Phys. Lett. B 194, 11 (1987); K. Muto, E. Bender, and H.V. Klapdor, Z. Phys. A 334, 187 (1989); A. Faessler, W. A. Kaminski, G. Pantis, and J. D, Vergados,Phys. Rev. C 43, R21 (1991).

[10] G. Pantis, F. Šimkovic, J.D. Vergados, and A. Faessler, Phys. Rev. C 53, 695 (1996); F. Šimkovic, J. Schwieger, M. Veselský, G. Pantis and A. Faessler, Phys. Lett. B 393, 267 (1997).

[11] F. Šimkovic, G. Pantis, J.D. Vergados, and A. Faessler, Phys. Rev. C 60, 055502 (1999). 
[12] J. Toivanen and J. Suhonen, Phys. Rev. Lett. 75, 410 (1995).

[13] J. Schwieger, F. Šimkovic, and A. Faessler, Nucl. Phys. A 600, 179 (1996).

[14] F. Šimkovic, A.A. Raduta, M. Veselský, and A. Faessler, Phys. Rev. C 61 , 044319 $(2000)$.

[15] R.V. Jolos, W. Rybarska-Nawrocka, Z. Phys. A 296, 73 (1980).

[16] J. Dukelsky, P.Schuck, Nucl. Phys. A 512, 466 (1990); D.S. Delion, J. Dukelsky, and P. Schuck, Phys. Rev. C 55, 2340 (1997); J. Dukelsky, G. Roepke, and P. Schuck, Nucl. Phys. A 628, 17 (1998); J. Dukelsky, P.Schuck, Phys. Lett. B 464, 164 (1999).

[17] A. Bobyk, W.A. Kamiński, and P.Zareba, Eur. Phys. J. A 5, 385 (1999); Nucl. Phys. A 669, $221(2000)$.

[18] F. Šimkovic, G. Pantis, and A. Faessler, Prog. Part. Nucl. Phys. 40, 285 (1998); Phys. Atom. Nucl. 61, 1218 (1998).

[19] A. Mariano and J.G. Hirsch, Phys. Rev. C 61, 054301 (2000). 


\section{TABLES}

TABLE I. Calculated nuclear matrix element $M_{\left\langle m_{\mathrm{ee}}\right\rangle}$ for $0 \nu \beta \beta$-decay of ${ }^{76} \mathrm{Ge}$ within the pn-QRPA, pn-RQRPA and the pn-SRQRPA and the corresponding upper limit on the effective neu-

trino mass $\left\langle m_{\mathrm{ee}}\right\rangle$ deduced from the experimental lower bound for a half-life $T_{1 / 2}^{0 \nu}\left({ }^{76} \mathrm{Ge}\right) \geq 5.7 \times 10^{25}$ years $[6]$. Here, m.s. I and m.s. II denote the model spaces which comprice the full $3-4 \hbar \omega$ and $2-4 \hbar \omega$ major oscillator shells, respectively.

\begin{tabular}{|c|c|c|c|c|c|c|c|}
\hline & \multirow[b]{2}{*}{$g_{\mathrm{ph}}$} & \multicolumn{2}{|c|}{ pn-QRPA } & \multicolumn{2}{|c|}{ pn-RQRPA } & \multicolumn{2}{|c|}{ pn-SRQRPA } \\
\hline & & m.s. I & m.s. II & m.s. I & m.s. II & m.s. I & m.s. II \\
\hline$M_{\left\langle m_{\mathrm{ee}}\right\rangle}$ & 0.8 & 2.76 & 1.66 & 3.15 & 2.80 & 0.72 & 0.65 \\
\hline$\left\langle m_{\mathrm{ee}}\right\rangle$ & & 0.28 & 0.46 & 0.24 & 0.27 & 1.1 & 1.2 \\
\hline$M_{\left\langle m_{\mathrm{ee}}\right\rangle}$ & 1.0 & 2.54 & 1.53 & 2.95 & 2.61 & 0.66 & 0.59 \\
\hline$\left\langle m_{\mathrm{ee}}\right\rangle$ & & 0.30 & 0.50 & 0.26 & 0.29 & 1.2 & 1.3 \\
\hline
\end{tabular}




\section{FIGURES}

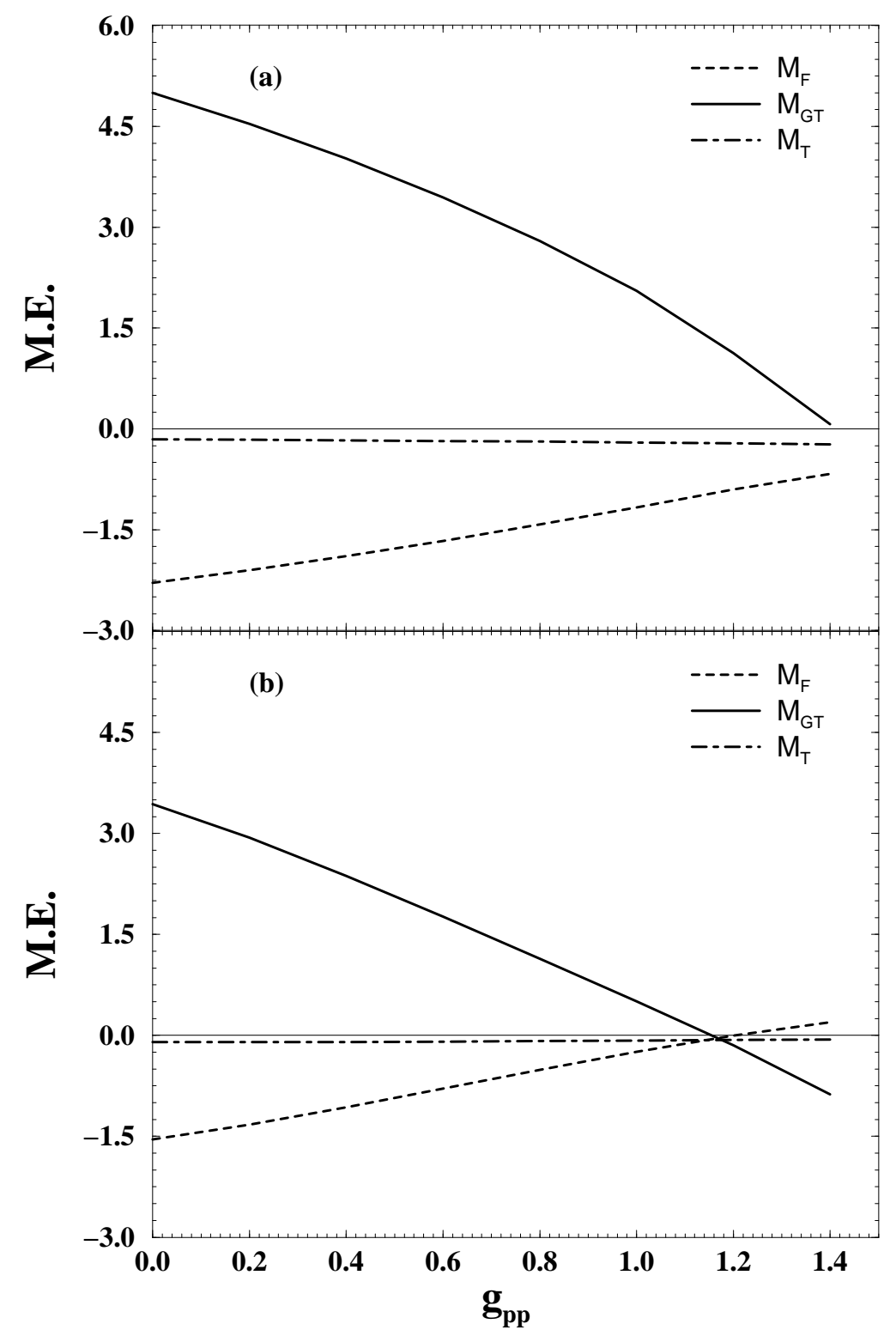

FIG. 1. The Fermi $\left(M_{F}\right.$, dashed line), the Gamow-Teller $\left(M_{G T}\right.$, solid line) and tensor $\left(M_{T}\right.$, dot-dased line) nuclear matrix elements for the $0 \nu \beta \beta$-decay of ${ }^{76} \mathrm{Ge}$ calculated within the pn-RQRPA (a) and pn-SRQRPA (b) are plotted as a function of the particle-particle coupling constant $g_{\mathrm{pp}}$. The 12-level model space II (the full $2-4 \hbar \omega$ major oscillator shells) was assumed and $g_{\mathrm{ph}}=1.0$ 


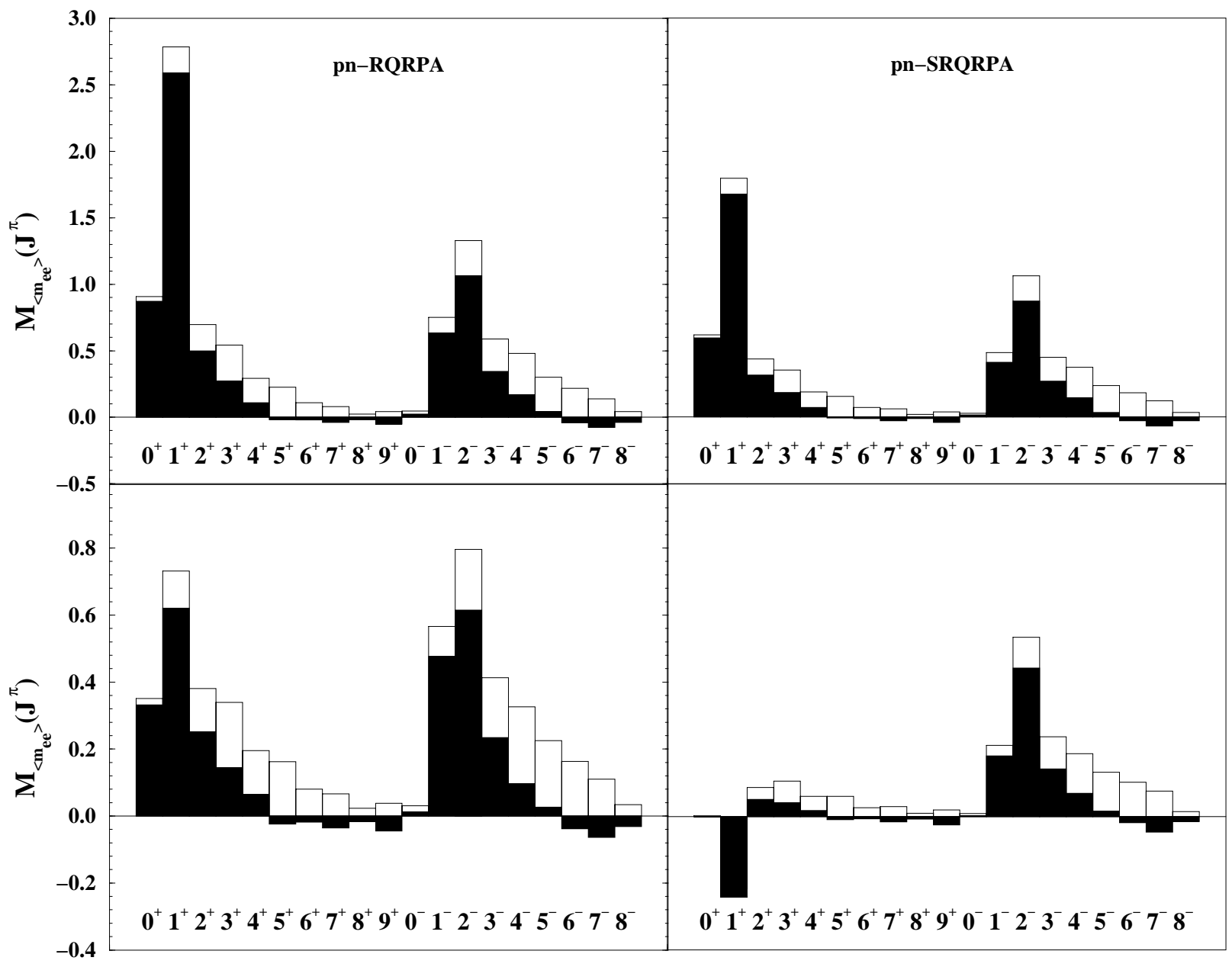

FIG. 2. The contributions of different multipolarities to the nuclear matrix element $M_{\left\langle m_{\mathrm{ee}}\right\rangle}$ for the $0 \nu \beta \beta$-decay of ${ }^{76} \mathrm{Ge}$ calculated within the pn-RQRPA (left figures) and pn-SRQRPA (right figures) without (open bar) and with (closed bar) consideration of the nucleon short-hand correlations (s.r.c.). The model space is the same as in Fig. 1 and $g_{\mathrm{ph}}=1.0$. The results in upper and lower graphs were obtained for $g_{\mathrm{pp}}=0.0$ and $g_{\mathrm{pp}}=1.0$, respectively. 


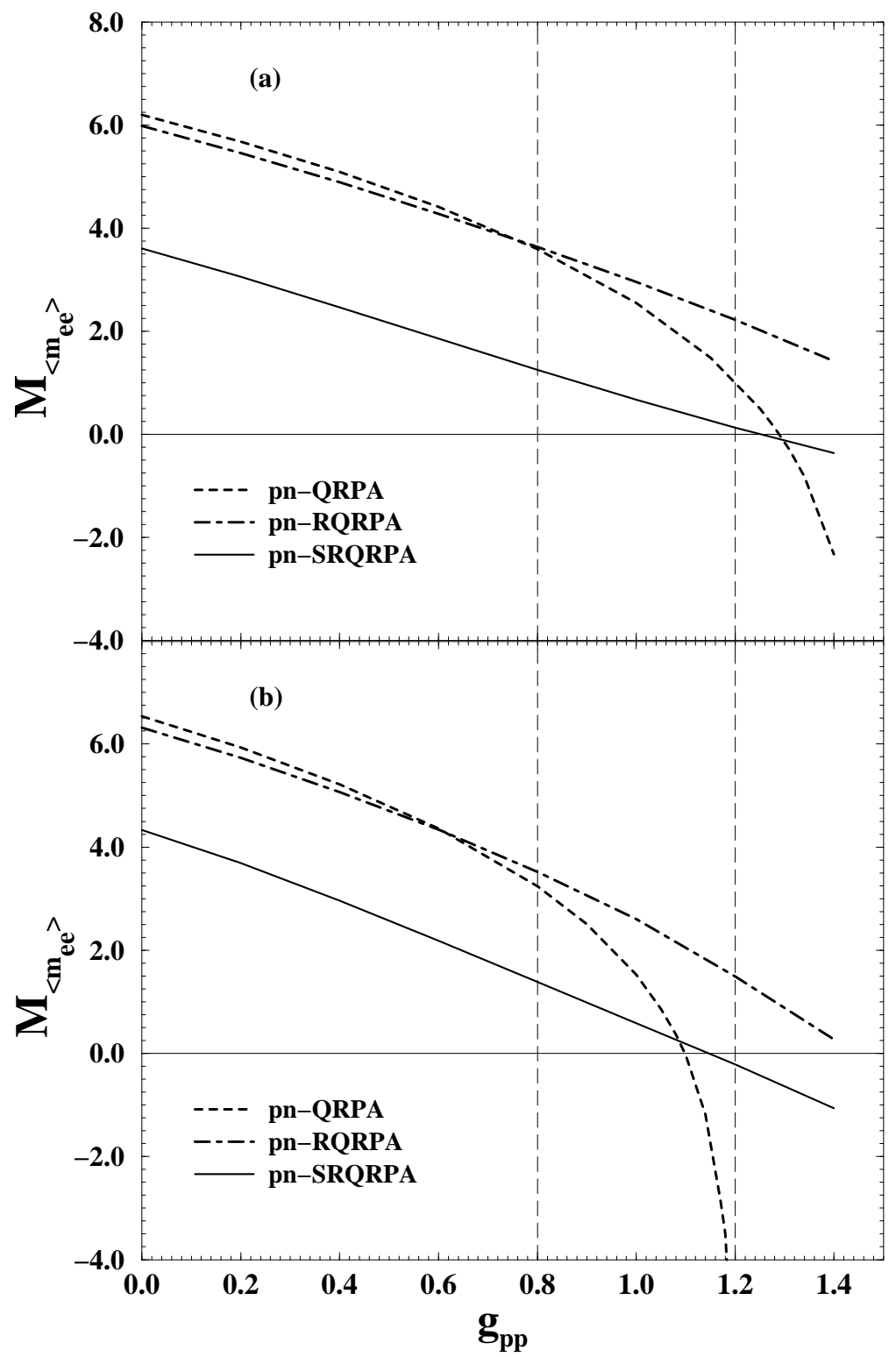

FIG. 3. The nuclear matrix element $M_{\left\langle m_{\mathrm{ee}}\right\rangle}$ for the $0 \nu \beta \beta$-decay of ${ }^{76} \mathrm{Ge}$ calculated within the pn-QRPA (dashed line), pn-RQRPA (dot-dashed line) and pn-SQRPA (solid line) in the framework

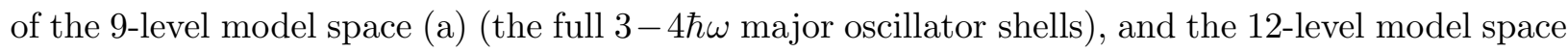
(b) (the full $2-4 \hbar \omega$ major oscillator shells) is plotted as a function of the particle-particle coupling constant $g_{\mathrm{pp}} \cdot g_{\mathrm{ph}}$ was taken to be unity. 